
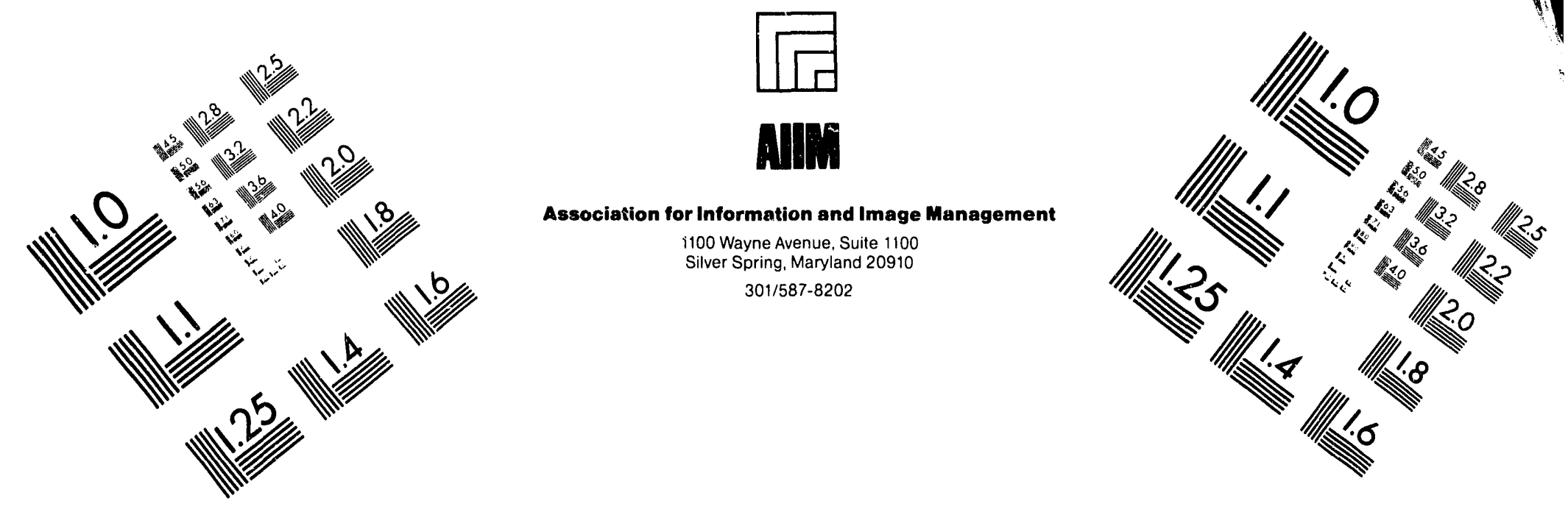

Centimeter

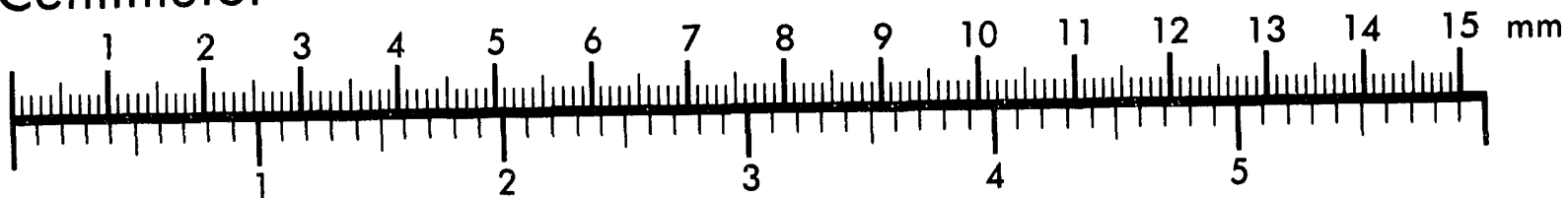
Inches
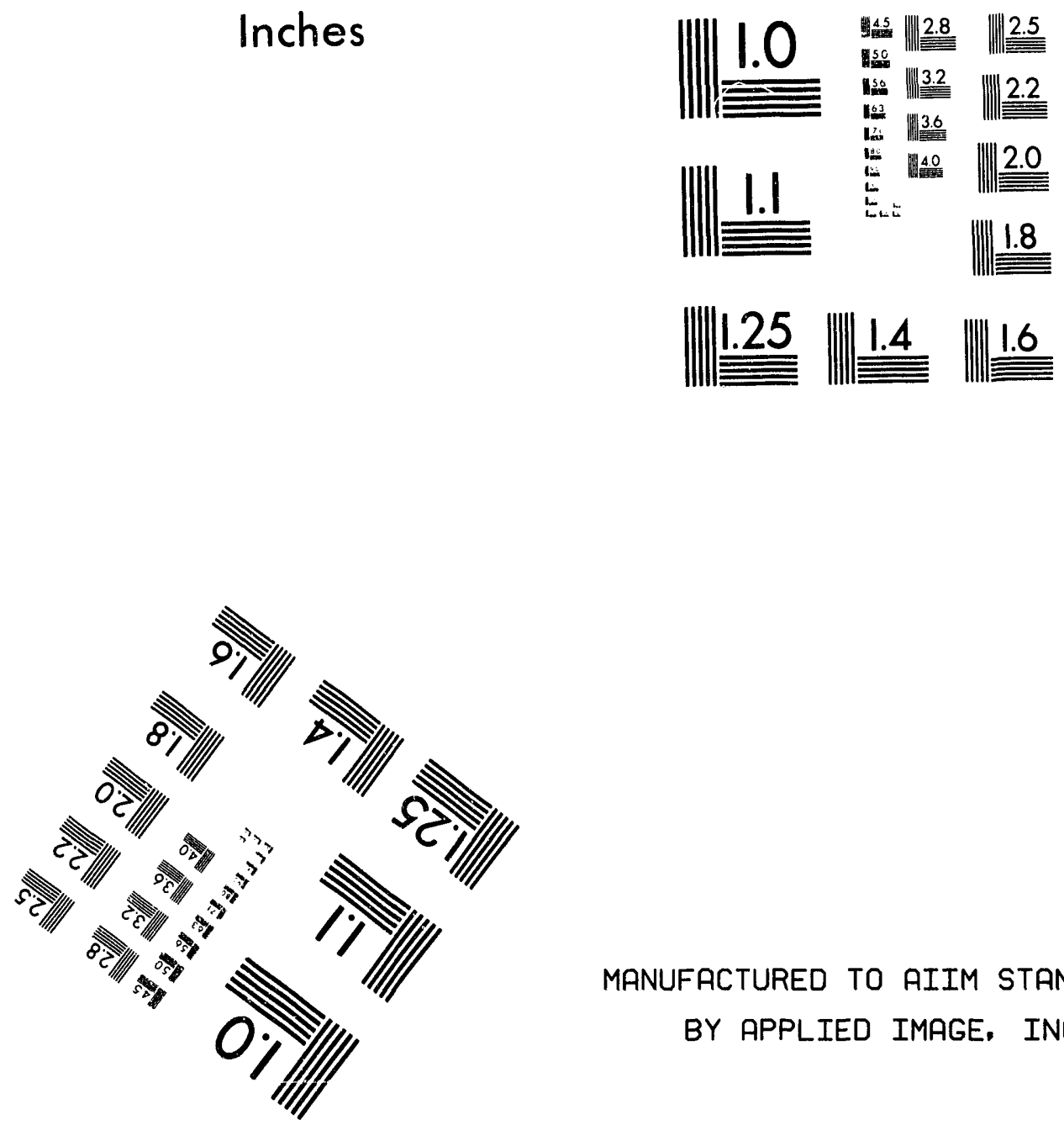

MANUFACTURED TO AIIM STANDARDS BY APPLIED IMAGE, INC.

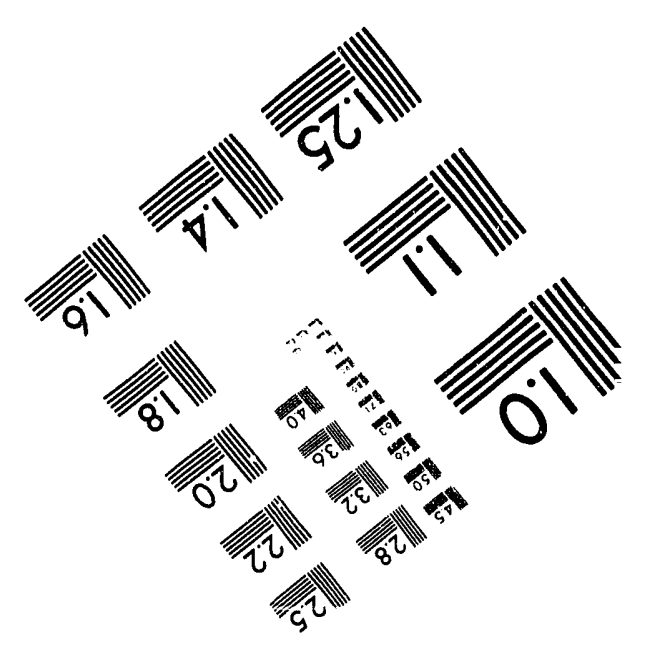



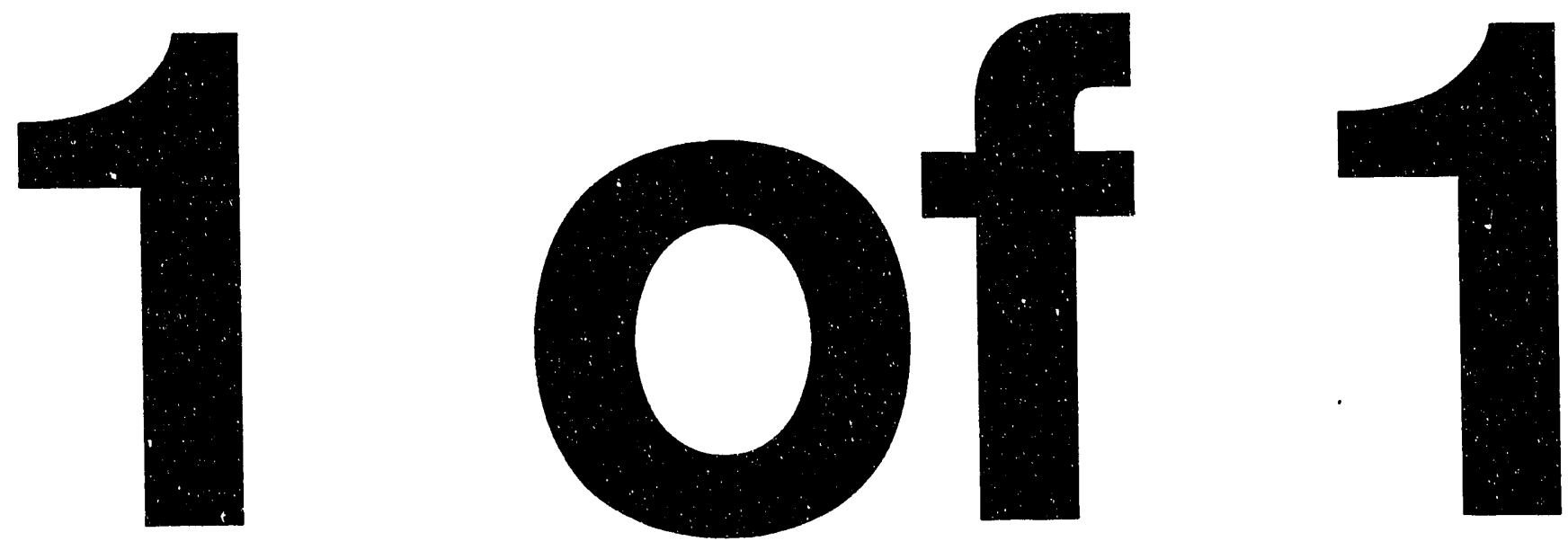
LBL-34052

UC-404

\title{
MAGNETIC MEASUREMENTS OF THE 5 METER QC SERIES QUADRUPOLES AT LAWRENCE BERKELEY LABORATORY
}

\author{
Paul Barale, ${ }^{1}$ B. Benjegerdes ${ }^{2}$, S. Caspi, ${ }^{2}$ M.I. Green, ${ }^{1}$ A. \\ Lietzke, ${ }^{2}$ R. Schermer, ${ }^{1}$ C. Taylor, ${ }^{2}$ and D. Van Dyke ${ }^{1}$ \\ 1Engineering Division* \\ ${ }^{2}$ Accelerator and Fusion Research Division \\ Lawrence Berkeley Laboratory \\ University of California \\ Berkeley, CA 94720 USA
}

MT-470

SC-MAG-409

Paper presented at the Fifth Annual International Industrial Symposium on the Super Collider (IISSC)

May 6-8, 1993

\footnotetext{
* This work was supported by the Director, Office of Energy Research, Office of High Energy and Nuclear Physics, Division of High Energy Physics, of the U.S. Department of Energy Under Contract No. DE-AC03-76SF00098.
} 


\title{
MAGNETIC MEASUREMENTS OF THE 5 METER QC SERIES QUADRUPOLES AT LAWRENCE BERKELEY LABORATORY
}

\author{
Paul Barale, ${ }^{1}$ B. Benjegerdes, ${ }^{2}$ S. Caspi, ${ }^{2}$ M.I. Green, ${ }^{1}$ A. Lietzke, ${ }^{2}$ \\ R. Schermer, ${ }^{1}$ C. Taylor, ${ }^{2}$ and D. Van Dyke ${ }^{1}$ \\ ${ }^{1}$ Magnetic Measurements Engineering Group* \\ 2Superconducting Magnet Group \\ Lawrence Berkeley Laboratory \\ Berkeley, CA 94720
}

\section{INTRODUCTION}

From May 1991 to September 1992, magnetic measurements were performed on six 5 meter prototype SSC quadrupoles designed and built at Lawrence Berkeley Laboratory (LBL). In addition, one of the quadrupoles was disassembled, reassembled and remeasured. The purpose of this paper is to review the magnetic measurements program and give an summary of some of the results of the magnet testing.

\section{THE MAGNETIC MEASUREMENT PROGRAM}

\section{The "MFM" Magnetic Measurement System}

The "Magnetic Field Measurement" (MFM) System is a general purpose rotating coil, harmonic analysis magnetic measurement system developed at LBL for measurement of the SSC $5 \mathrm{~m}$ prototype quadrupoles. The system has the following significant features:

1. Externally driven tangential measuring coils with quad and dipole bucking coils

2. External optical encoder - angular position to $43.75 \mu$ radians absolute

3. Integrated induced voltage measured using digital integrator system

4. Analog bucking to obtain high resolution harmonic content of magnetic field

5. UNIX workstation and VME crate in host-target configuration - all real time data acquisition tasks in dedicated VME crate, operator interface, data analysis and display, and other non-real time tasks in UNIX workstation.

The MFM control, acquisition and analysis software packages were all written in $\mathrm{C}$ and developed at LBL. Acquired data is corrected for linear drift, rotated into a 'standard' frame of reference (south pole at $45^{\circ}$ when viewed from the non-lead end), and corrected for differences between search coil axis of rotation and the magnetic center of the magnet. In addition, all warm measurements are taken with both positive and negative currents and the results averaged, to remove effects of external fields (e.g. earth's field, iron remnant

\footnotetext{
* This work was supported by the Director, Office of Energy Research, Office of High Energy and Nuclear Physics, Division of High Energy Physics, of the U.S. Department of Energy Under Contract No. DE-AC0376 SF00098.
} 
magnetization in yoke, etc.).

\section{The Measurements Program}

All magnets were measured both at room and cryogenic temperatures. Our typical test program includes the following:

\section{Room Temperature $\quad$ Cryogenic}

1. Uniform field region, $\pm 14 \mathrm{~A} \quad 1$. Uniform field region, $0->6.6->0 \mathrm{kA}$

2. Axial scan, $\pm 14 \mathrm{~A}$

2. Axial scan, $3 \mathrm{kA}$

3. Harmonic decay, $3 \mathrm{kA}$

Operational details of these tests can be found elsewhere ${ }^{1}$.

Both the measurement hardware and software were under development during the measurement of QCC401 and QCC402, consequently the testing of these two magnets was not as extensive as the remainder of the group. In addition, scheduling and research emphasis dictated somewhat the test selection from magnet to magnet.

\section{RESULTS}

\section{Axial Scan}

The axial scan data provided the most interesting and useful results. Figure 1 illustrates one unexpected effect noted in all cold axial scans. As can be clearly seen, the b5 multipole (first allowed harmonic of b1, the quadrupole) is distorted for positions $<140 \mathrm{~cm}$ and $>400 \mathrm{~cm}$, leaving a "uniform field" region of $\sim 260 \mathrm{~cm}$. Centered in these distorted regions are the strain gauge packs, whose influence is integrated over a wide region by the 1 meter long measuring coil. A similar effect can be seen in the b9 (second allowed) harmonic. The effect is not seen in the warm axial scans.

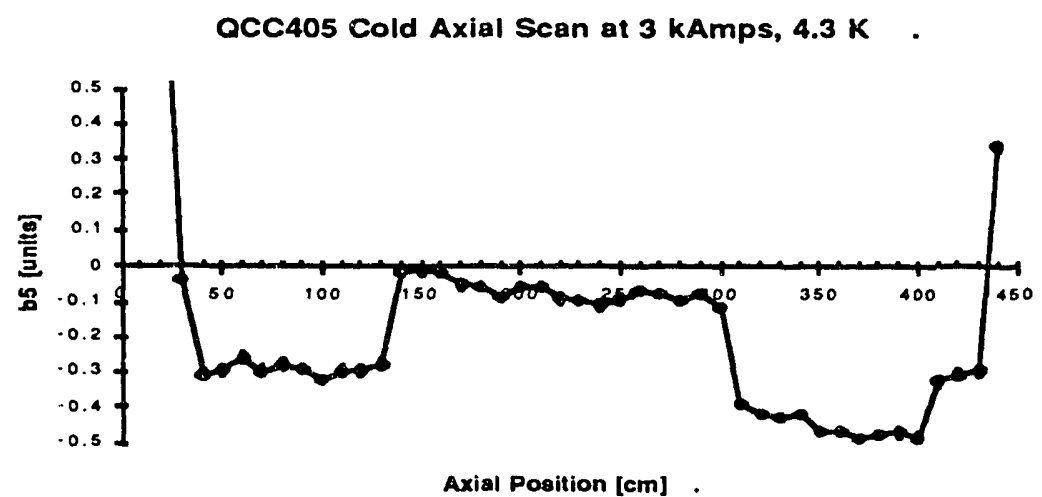

Figure 1. b5 vs Axial Position for QCC405 Cold Axial Scan

\section{Multipole Summary}

Table 1 summarizes the average cold axial multipoles for magnets QCC402 through QCC406. The data quoted are the average values over all axial scans and can be considered representative of the "uniform field" region of each magnet at 3000 amps. Detailed explanation of the analysis used to obtain these results are available ${ }^{2}$.

Table 1. Multipcle Summary [units]

\begin{tabular}{lcccccccc}
\hline & $\mathrm{a} 2$ & $\mathrm{a} 3$ & $\mathrm{a} 4$ & $\mathrm{a} 5$ & $\mathrm{a} 6$ & $\mathrm{a} 7$ & $\mathrm{a} 8$ & $\mathrm{a} 9$ \\
\hline QCC402 & -0.724 & 0.553 & -0.091 & 0.222 & 0.012 & 0.028 & -0.057 & 0.065 \\
QCC403 & 1.296 & -0.832 & 0.167 & 0.010 & -0.008 & -0.001 & 0.013 & -0.013 \\
QCC404 & 1.552 & 0.103 & 0.126 & -0.115 & 0.015 & 0.031 & 0.029 & -0.001 \\
QCC405 & -0.485 & 0.280 & 0.147 & -0.046 & 0.036 & 0.023 & 0.005 & 0.000 \\
QCC405A & -0.456 & 0.414 & 0.174 & -0.043 & 0.034 & 0.017 & 0.005 & 0.002 \\
QCC406 & -0.662 & 0.467 & -0.236 & 0.005 & 0.004 & 0.025 & 0.003 & 0.009 \\
SSC Spec. & 2.696 & 1.550 & 0.641 & 0.738 & 0.209 & 0.240 & 0.276 & 0.317 \\
& & & & & & & &
\end{tabular}




\begin{tabular}{ccccccccc}
\hline & $\mathrm{b} 2$ & $\mathrm{~b} 3$ & $\mathrm{~b} 4$ & $\mathrm{~b} 5$ & $\mathrm{~b} 6$ & $\mathrm{~b} 7$ & $\mathrm{~b} 8$ & $\mathrm{~b} 9$ \\
\hline QCC402 & -0.284 & -0.041 & -0.195 & -1.082 & 0.086 & -0.072 & 0.002 & 0.102 \\
QCC403 & 1.189 & -0.155 & 0.058 & $-0.85-$ & -0.025 & 0.041 & 0.101 & 0.234 \\
QCC404 & -0.694 & -1.009 & 0.023 & -0.352 & -0.064 & -0.014 & 0.023 & 0.147 \\
QCC405 & -0.184 & -0.111 & 0.195 & -0.082 & 0.044 & -0.004 & 0.008 & 0.091 \\
QCC405A & -0.151 & 0.306 & 0.215 & 0.004 & 0.043 & -0.016 & -0.002 & 0.087 \\
QCC406 & 0.148 & 0.128 & 0.162 & -1.486 & 0.028 & 0.006 & 0.001 & 0.177 \\
SSC Spec. & 2.696 & 1.550 & 0.641 & 1.680 & 0.209 & 0.240 & 0.276 & 0.776
\end{tabular}

SSC Spec. in this case is defined as 1 Systematic +1 RMS for each multipole 3 .

\section{Warm-Cold Correlation}

Multipole data obtained from warm and cryogenic axial scans were examined for correlation. Axial scan data was used to gain enough points to achieve statistical significance and because some of the multipoles were sensitive to axial positioning. Data distorted by the strain gauges were excluded. Figures 2 below illustrate the correlation for the first allowed multipoles. These multipoles were not corrected for persistent current magnetization effects in the cold measurements (expected to be -0.08 units for b5 and 0.004 units for b9). The data for these multipoles is clustered around points which lie on the diagonal, suggesting little geometric change during cooldown. All the low order multipoles (b2 through a4) exhibit some degree of correlation. In the higher order multipoles, the data lies on a line parallel to the $\mathrm{x}$-axis, suggesting that these are dominated by noise in the warm measurements. ${ }^{2}$

\section{QC Series W-C Correlations}

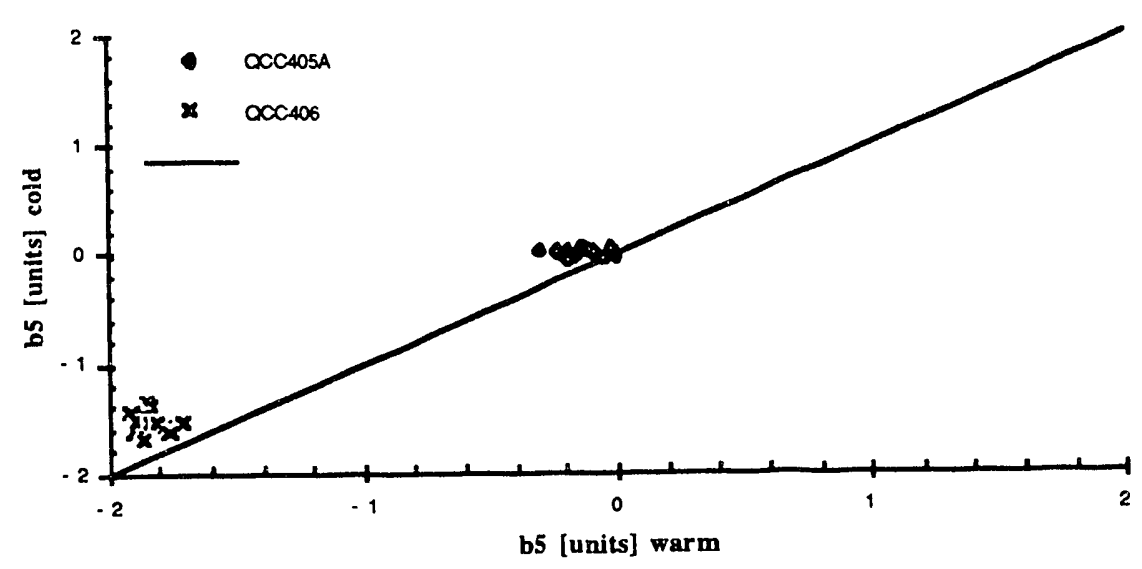

QC Series W-C Correlations

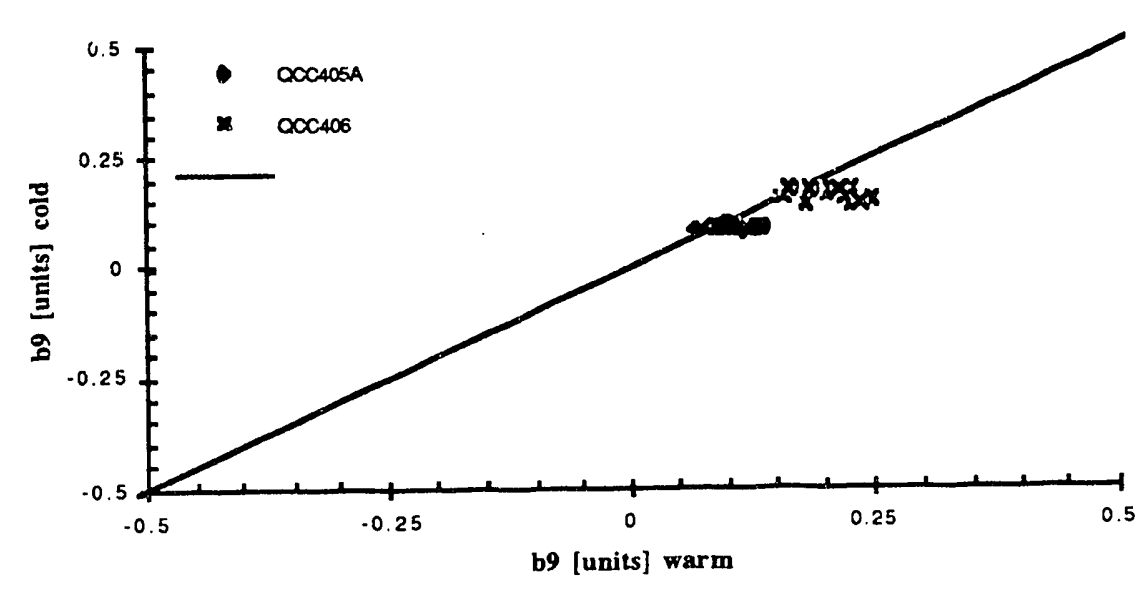

Figure 2. Warm-Cold Correlation of Allowed Multipoles 


\section{Injection Decay}

None of the QC series quadrupoles exhibited significant injection current first allowed harmonic decay, in part due to small b5 values for the series (except for QCC406, where a significant b5 was deliberately designed in). However, even in QCC406, the decay is on the order of a tenth of a unit over an hour - considerably smaller than decays seen in the SSC dipole first harmonic. Decay for magnets QCC401 through QCC404 are not included in Figure 3 (below) as they are dominated by power supply instabilities. These instabilities were reduced for QCC405 and QCC406, and further corrected prior to QCC405A.

QC Series Quadrupoles - Injection Current Decay

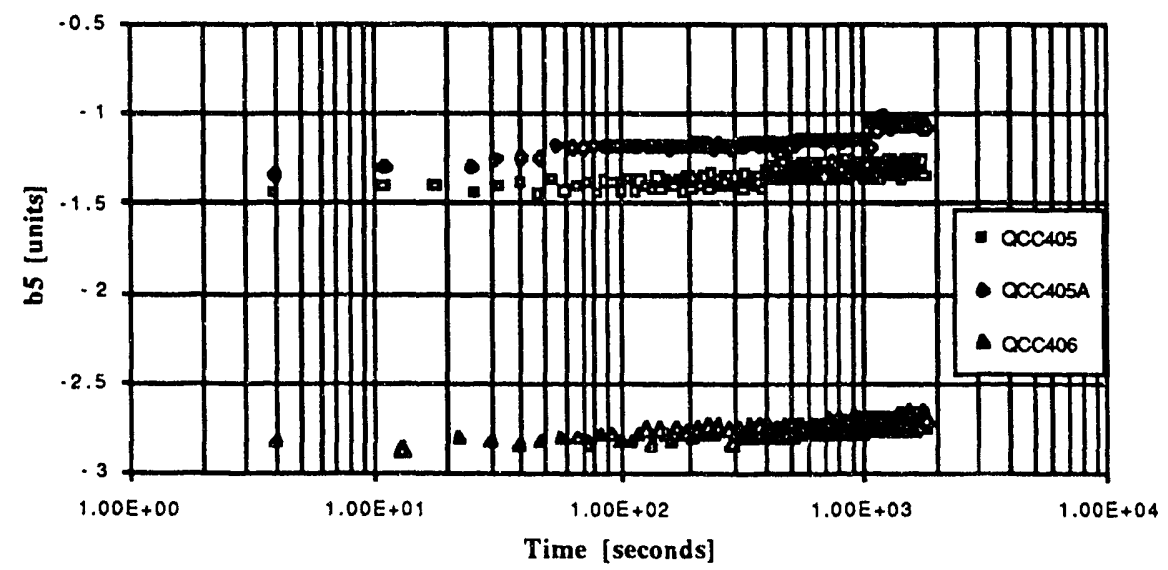

Figure 3. b5 Decay at Injection Current

\section{SUMMARY}

From the magnetic measurements performed on the QC series quadrupoles at $\mathrm{LBL}$, it is clear that this design easily meets the SSC specifications on field quality. In addition, multipole decay during injection should not be a significant problem. Finally, it appears that there may be sufficient warm-cold correlation of the lower multipoles to allow for acceptance/rejection decisions to be based on warm measurements, particularly if a more sensitive coil, or higher currents are used for the warm measurements. On the down side, there can be significant local variations in field and field quality that will show up only on axial scans. These are, however, local variations and the effects are minimized when one considers the magnet integral field.

Overall, the QC series of quadrupoles and the related magnet measurement program have to be considered a success, from the standpoint of field quality.

\section{REFERENCES}

1. P.J. Barale. "Typical Magnetic Measurements of 5 Meter SSC Quadrupoles for the LBL Superconducting Magnet Group," Lawrence Berkeley Lab LBID 1838, SC-MAG-358, MT-441 (March 1992).

2. Norihito Ohuchi. "Z-scan Magnetic Measurements of LBL Quadrupole Magnets (QCC402-406)," SSC Laboratory, Test and Data Management MD-TA-251 (March 1993).

3. SSC Internal Note Document Number M80-000007, SSC Laboratory (May 1991). 

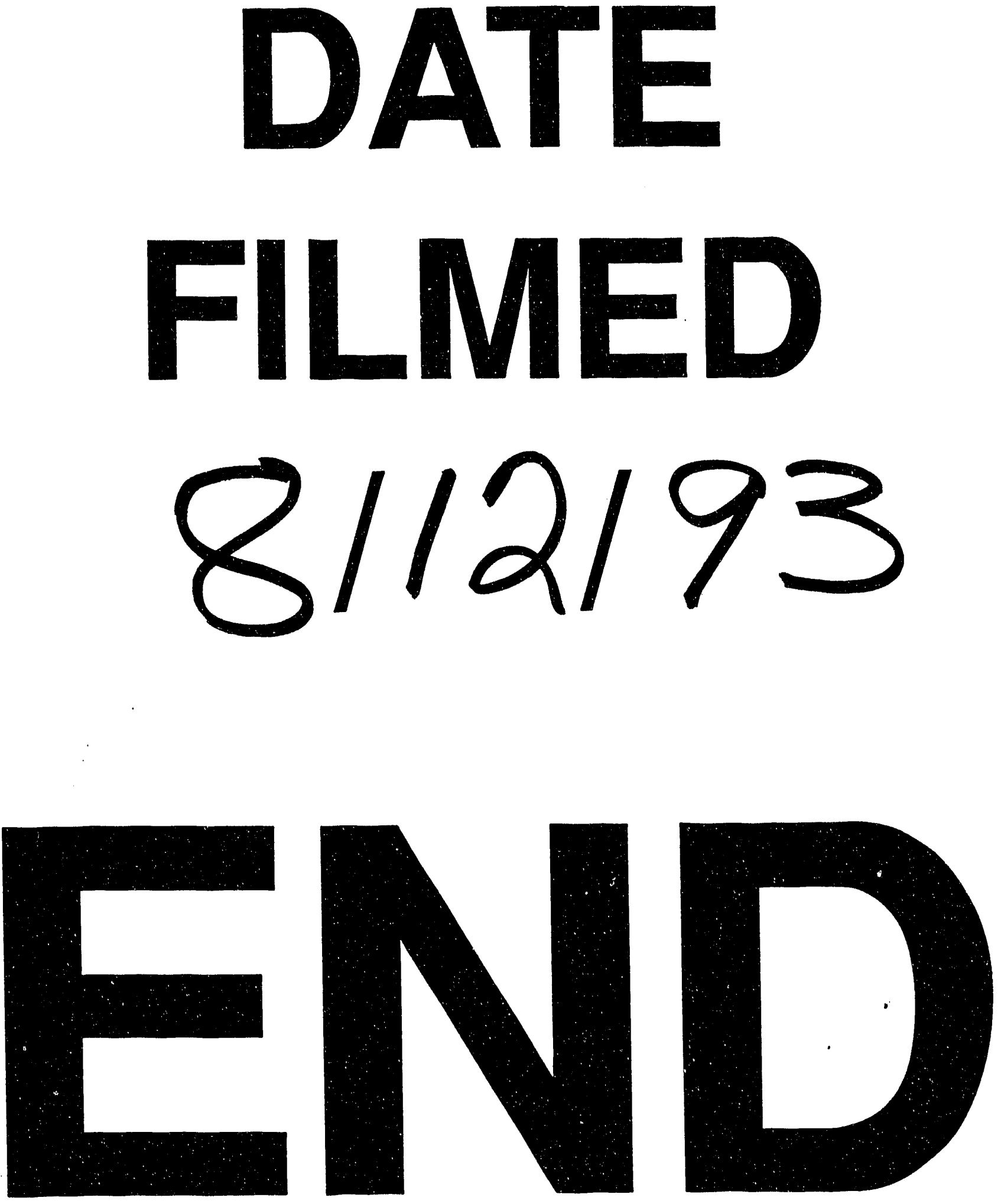
